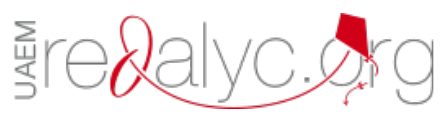

Centro Sur

ISSN: $2600-5743$

compasacademico@icloud.com

Grupo Compás

Ecuador

\title{
Sendero Turístico-Ecológico para la Comuna Palmar, Parroquia Colonche
}

Balladares Pongillo, Karen; López Franco, Melvin

Sendero Turístico-Ecológico para la Comuna Palmar, Parroquia Colonche

Centro Sur, vol. 1, núm. 1, 2017

Grupo Compás, Ecuador

Disponible en: http://www.redalyc.org/articulo.oa?id=588861699001

Esta obra está bajo una Licencia Creative Commons Atribución-NoComercial-SinDerivar 4.0 Internacional. 


\title{
Sendero Turístico-Ecológico para la Comuna Palmar, Parroquia Colonche
}

\author{
Tourist-Ecological Trail for the Palmar Commune, Colonche \\ Parish \\ Karen Balladares Pongillo karen.balladaresp@ug.edu.ec \\ Universidad de Guayaquil, Ecuador \\ http://orcid.org/0000-0002-7235-1147 \\ Melvin López Franco melvinleonardo@hotmail.com \\ Universidad de Guayaquil, Ecuador \\ http://orcid.org/0000-0003-3065-215X
}

Centro Sur, vol. 1, núm. 1, 2017

Grupo Compás, Ecuador

Recepción: 16 Junio 2016 Aprobación: 26 Octubre 2017

Redalyc: http://www.redalyc.org/ articulo.oa?id=58886169900 1

\section{BY-NC-ND}

Resumen: El objetivo de este estudio es desarrollar el turismo ecológico por medio del diseño y estudio de un sendero turístico - ecológico para los turistas de la comuna Palmar. Los objetivos específicos son: analizar los referentes teóricos de vías de acceso peatonal y vehicular, determinar la correcta ubicación y recorrido del sendero turísticoecológico, elaborar un diseño sustentable de un sendero turístico que cumpla con normas de desarrollo sostenible y definir un modelo de gestión que garantice la sustentabilidad del proyecto.

Palabras clave: Turismo, Sendero, Santa Elena.

Abstract: The aim of this study is to develop ecological tourism through the design and study of a tourist trail - ecological for tourists Palmar commune. The specific objectives are: to analyze the theoretical framework of routes of pedestrian and vehicular access, determine the correct location and path of the tourist-ecological path, develop a sustainable design of a tourist trail that meets standards of sustainable development and to define a management model to ensure the sustainability of the project.

Keywords: Tourism, Trail, Santa Elena.

Abstract: The aim of this study is to develop ecological tourism through the design and study of a tourist trail - ecological for tourists Palmar commune. The specific objectives are: to analyze the theoretical framework of routes of pedestrian and vehicular access, determine the correct location and path of the tourist-ecological path, develop a sustainable design of a tourist trail that meets standards of sustainable development and to define a management model to ensure the sustainability of the project.

Keywords: Tourism, Trail, Santa Elena.

\section{INTRODUCCIÓN}

El objeto de estudio del presente trabajo investigativo es la red vial del Ecuador, que es considerado un pilar básico para el fomento de la productividad basada en los principios de equidad, equivalencia, excelencia, sostenibilidad ambiental y competitividad, haciendo posible el cumplimiento del plan nacional del desarrollo y los principios del Buen Vivir o Sumak Kawsay. (Ministerio de Transporte y Obras, 2013)

Con lo que respecta a la accesibilidad Vehicular-Peatonal, se debe precisar que se puede ingresar a la comuna Palmar por medio de la vía de primer orden que va desde Santa Elena hasta la Provincia de Esmeralda, Ruta del Spondyllus (Ruta del Sol) y en el desvió Colonche - Palmar existe 
una vía de segundo orden cuya carretera es asfaltada. Dentro de la comuna existen vías de tercer orden que comunican el centro con la playa y los sitios turísticos como son el manglar, el cerro María Guare, Santuario de nuestra Señora de Fátima, entre otros.

El problema de la comuna es la falta de los diferentes accesos a los sitios turísticos, tales como el manglar, el cerro María Guare, Santuario de nuestra Señora de Fátima, entre otros, provocando que los turistas busquen ayuda a los habitantes del sector ya que no existen puntos de información.

La deficiencia en los accesos a los sitios turísticos en la comuna Palmar de la Parroquia Colonche del Cantón Santa Elena, ha sido provocada por la carencia de mantenimientos y deterioro por el tiempo de uso de las vías. La afluencia vehicular elevada en la zona, así como la falta de mantenimiento ha llevado al deterioro de las vías.

Entre las causas que originan el problema de investigación es la deficiencia en la aplicación de normas turísticas y del buen vivir debido al bajo interés de involucramiento en la aplicación de normas turísticas y del buen vivir. Por lo que la investigación se centra en la siguiente pregunta científica: ¿Con el mejoramiento de acceso vial en la comuna Palmar de la Parroquia Colonche del Cantón Santa Elena se logrará tener un incremento turístico?

Palmar cuenta con atractivos naturales y culturales se beneficiará económicamente mediante trabajos indirectos como: restaurantes, tiendas, artesanías. También se capacitará a los habitantes en técnicas de guía con el fin de que los turistas reciban información real y clara sobre estos atractivos. Además, los turistas podrán disfrutar, conocer, observar de la flora, fauna, mar y hermosos paisajes, al igual que los estudiantes de turismo y afines a la misma, ya que dispondrán de información valiosa sobre senderos sostenibles.

El objetivo general es desarrollar el turismo ecológico por medio del diseño y estudio de un sendero turístico - ecológico para los turistas de la comuna Palmar. Los objetivos específicos son: analizar los referentes teóricos de vías de acceso peatonal y vehicular, determinar la correcta ubicación y recorrido del sendero turístico-ecológico, elaborar un diseño sustentable de un sendero turístico que cumpla con normas de desarrollo sostenible y definir un modelo de gestión que garantice la sustentabilidad del proyecto.

Sobre la base de los fundamentos teóricos la red vial y accesibilidad vehicular peatonal y de las categorías analíticas como la afluencia vehicular elevada en la zona, carencia de socialización en programas turísticos hacia la zona y el bajo interés en involucrarse en aplicación de normas turísticas y del buen vivir se concluye la elaboración de un diseño sustentable de un sendero turístico que cumpla con normas y categorías viales que se definen en la ordenanza territorial del cantón Santa Elena.

Según la Ordenanza de Ordenamiento Territorial (2006), la vialidad urbana se organiza atendiendo categorías viales que se definen, según su jerarquía y rol, de la siguiente manera: 
Red Vial Fundamental. - Está constituida por vías primarias, las qué por sus características de sección y trazado, o por intensidad de tráfico, comunican y dan acceso a grandes zonas de la ciudad o brindan acceso vehicular a la misma. Se identifican con el código 05 .

Constituyen categorías y componentes de la Red Vial Fundamental, los siguientes ejes viales:

a) Autopistas (V1), con derecho de vía de noventa a cien metros (90-100 m.), y con accesos vehiculares controlados mediante facilidades de tráfico.

b) Vías Expresas (V2), con derecho de vía de setenta a noventa metros (70-90 m.)

c) Vías Arteriales (V3), con derecho de vía de treinta a setenta metros (30- $70 \mathrm{~m})$.

d) Rutas Troncales de Transporte (VT), con derecho de vías variables de acuerdo al proyecto específico. A pesar de no contar con características físicas adecuadas, son excepcionalmente reconocidas en esta Ordenanza, como parte de la Red Vial Fundamental:

e) Pares Viales (PV), sistemas de vialidad binaria, qué mediante ejes paralelos, y de sentidos de tráfico opuestos entre sí, facilitan el acceso a áreas importantes de la ciudad o a otros componentes de la red vial primaria.

La infraestructura vial en el Ecuador, ha mantenido una historia de afectaciones constantes, como paralizaciones y colapso de puentes y caminos, generadas tanto por el riesgo sísmico cuanto por los factores climáticos a los que por décadas los Gobiernos han tenido que afrontar con soluciones inmediatistas y onerosas para el erario nacional, sin ningún soporte tecnológico que garantice una seguridad adecuada para el desarrollo.

Las afectaciones de la red vial antes señaladas a su vez, de forma directa, han incidido negativamente al proceso de desarrollo económico y productivo del Ecuador, fomentando la pobreza e imitando el acceso a bienes, productos y servicios vitales garantizados por la Constitución. Las regulaciones técnicas del MTOP (MOP-001-F y MOP-001-E), generadas en 1974 han contribuido tibiamente en solucionar los aspectos antes mencionados, en 1993 fueron actualizadas con mínimos cambios por la Pontificia Universidad Católica del Ecuador. A través de Acuerdos Ministeriales se ha tratado de homologar y regular aspectos no contemplados en las regulaciones antes señaladas y la formulación de Normas Interinas de 1999 Ex CORPECUADOR, ha llegado a constituir una guía técnica referencial para reducir las probabilidades de fallas de las obras de reconstrucción de la red vial.

La red vial a implementarse en las comunas, deberán tener como soporte el estudio y desarrollo de los diferentes aspectos:

Aplicación de normas de tráfico que contemplen la provisión obligatoria de espacios para circulación y aparcamiento vehicular público (para el servicio de las áreas residenciales, y para acceso de los sistemas de emergencia y de visitantes en general), así como para la circulación peatonal y cualquier otra modalidad de circulación, todo ello vinculado y 
referido a la elaboración de un estudio que en el marco de lo establecido en la Ley Orgánica de Transporte Terrestre Tránsito y Seguridad Vial en vigencia, determine las demandas con relación a estos servicios, generadas por las densidades poblacionales y edificatorias a desarrollarse en el proyecto. (M.I. Municipalidad de Guayaquil, 2011)

La capacidad, secciones, funciones de las vías producto de estudio anterior deberá considerar adicionalmente los parámetros establecidos en los cuadros denominados Características de Diseño del Sistema Vial según Normas de Vialidad y Tráfico y su relación con usos de suelo, así como el de Normas de Diseño Geométrico para la Red Vial Fundamental y Secundaria, así como aspectos relacionados con Especificaciones Técnicas, Diseño de Hormigón, Perfiles, Pendientes de la red vial a proponer.

Independientemente de los resultados de los estudios anteriormente relacionado se deja establecido qué en toda vía vehicular, independiente de su sección mínima de calzada, se deberá prever en el caso de las aceras, cuando estas sean utilizadas para el paso subterráneo de más de dos (2) de las redes de servicio público y/o privadas (sanitarios, eléctrico, telefónico, de datos, etc) una sección no menor de $2.5 \mathrm{ml}$.

Según (Ruiz Ballesteros \& Solis Carrión, 2007, pág. 29) “El turismo en Ecuador inicia su desarrollo moderno en los años 50 del siglo XX, es principalmente en las tres últimas décadas, en que empieza a consolidarse como actividad económica y socio - cultural de importancia”.

En 1992 en el Gobierno de Sixto Duran Ballén muestra un interés y realiza la creación de Ministerio de Turismo y a partir de esa fecha Ecuador empieza a dar sus primeros pasos para explotación de sus atractivos y hacerlos conocer al mundo. El ex Ministro Ehlers destacó que "Ecuador trascendió fronteras para posicionarse como el primer destino para turistas extranjeros en América Latina, de esta manera se logró que en el 2012 nuestro país participe en 55 ferias turísticas internacionales, mientras que en el 2006 apenas se llegaba a siete por año". (Ministerio de Turismo del Ecuador, 2013)

Hoy el turismo representa la cuarta actividad económica de mayor ingreso después del petróleo banano, el camarón y otros elaborados productos marítimos en el año en el 2012 al Ecuador ingresó 1.032,7 millones de dólares por año aumentado en el 2013 a 1.251,3 por esto se lo coloca como uno de los principales sectores productivos del país. (Ministerio de Turismo del Ecuador, 2014)

En la actualidad el Gobierno está muy convencido que en el sector turístico está la clave para la transformación de la matriz productiva del país en la cual se quiere convertir a cada uno de los sitios turísticos del Ecuador en destinos de excelencia porque de cada turista que ingresa al país representa una fuente de empleo para el turismo por tanto el Gobierno ha cuadruplicado el presupuesto que tenía estimado para este sector.

En el 2012, el presupuesto asignado para el Ministerio de Turismo fue de 40 millones de dólares, desde este año y hasta el 2017 se incrementará a 
150 millones anuales. (Andes Agencia publicitaria de noticias del Ecuador y Suramerica, 2013)

Esta comuna es conocida como el puerto de la parroquia Colonche, entre las actividades económicas a las que sus habitantes se dedican en su mayoría es a la pesca artesanal. Entre los atractivos turísticos que posee se encuentra: el manglar, la playa con su rompeolas, el santuario Nuestra Señora de Fátima, playa Rosada, entre otras. Entre la gastronomía de la comuna se encuentra platos típicos con mariscos recién salidos del mar: el arroz con pescado, ceviches de mariscos, ensaladas de mariscos y arroz con menestra y carne asada.

Entre las cooperativas de la comuna se posee la siguiente información: Cooperativa De Producción Pesqueras, Virgen Del Carmen, Cooperativa De Producción Pesquera, Artesanal Palmar, Plan de Desarrollo y Ordenamiento Territorial del Gobierno Autónomo Descentralizado de Colonche 2015, Asociación De Pescadores "El Palmar" (Changueros De Palmar)

Dentro de estas tres cooperativas existen varias actividades de pesca, originalmente todos los socios eran pescadores artesanales, pero en los últimos años se han dedicado a la pesca a mayor escala como son los barcos chinchorreros. Un porcentaje menor se mantiene con los artes de pesca artesanales como las redes camaroneras, panpaneras y de fondo. La última llamada asociación de Larveros quienes originalmente se formaron con el auge de la pesca de larvas de camarón, han optado por un nuevo arte de pesca conocida como la CHANGA, aunque no es legal en nuestra provincia se está gestionando con los organismos pertinentes para que se permita este tipo de pesca. Mediante un proyecto del estado, esta última asociación junto a la Fundación Neo juventud acogieron el proyecto de cultivo de ostras. Se cuenta con lugares aptos para brindar a los turistas hospedajes: Hostería marina 1 y 2, Hostería Puerto Palmar, Segovita beach house, Palmar y Regalo de Dios.

\section{MATERIALES Y MÉTODOS}

La metodología cualitativa permite entender cómo los participantes de una investigación perciben los acontecimientos. La variedad de sus métodos, como son: la fenomenología, el interaccionismo simbólico, la teoría fundamentada, el estudio de caso, la hermenéutica, la etnografía, la historia de vida, la biografía y la historia temática, reflejando la perspectiva de aquel que vive el fenómeno, es decir, del participante que experimenta el fenómeno. El uso de esta aproximación es de carácter inductivo y sugiere que, a partir de un fenómeno dado, se pueden encontrar similitudes en otro, permitiendo entender procesos, cambios y experiencias.

Según Hernández Sampieri y colaboradores aborda la visión cualitativa de manera fascinante, a través de ejemplos que facilitan la asimilación de las etapas esenciales de la investigación.

Yin (1989:29-36) propone una manera de pensamiento de diseño de la investigación refiriéndose a cinco componentes especialmente importantes: 
- Las preguntas de investigación

- Las proposiciones teóricas

- La(s) unidad(es) de análisis

- La vinculación lógica de los datos a las proposiciones

- Los criterios para la interpretación de los datos

Las preguntas de investigación y las proposiciones teóricas servirán de referencia o punto de partida para la recolección de los datos desde los distintos niveles de análisis del caso(s), y para el análisis posterior de los mismos. Pues tanto las preguntas de investigación como las proposiciones teóricas contienen los constructos (conceptos, dimensiones, factores o variables) de los cuales es necesario obtener información.

Por lo tanto, se debe proceder a presentar la forma como se recolectará la información relacionada con los constructos; es decir, explicitar tanto las diversas fuentes de las cuales se obtendrá como los instrumentos que han de utilizarse para la recolección de la misma, y posteriormente derivar la vinculación lógica de los datos obtenidos a dichas proposiciones.

Finalmente se presentarán los resultados de la investigación a través de una serie de conclusiones que conducirían al fortalecimiento de las teorías o de los enfoques insertos en el marco teórico de la investiga

En la República del Ecuador, a nivel de carreteras de segundo orden, salvo pocas excepciones, la gestión vial es precaria. La construcción de las carreteras, qué si bien se han ejecutado siguiendo especificaciones similares a las controladas por el actual Ministerio de Transporte y Obras Públicas, no han proporcionado información para la futura gestión y programación de intervenciones. La mayoría de las administraciones seccionales en el Ecuador, no disponen de un sistema de información completo de las redes viales que administran; por ello, con frecuencia no pueden responder con oportunidad y exactitud cuándo se requiere conocer sobre: la extensión, jerarquía y finalmente su condición y requerimientos de intervención para una adecuada gestión del mantenimiento. El problema que se ha observado en las carreteras de segundo orden del Ecuador es la generación de un deterioro excesivo de los elementos viales, porque no se implementa un sistema de control, inventario, monitoreo y de provisión de datos para futuros planes y programas de intervención, aplicable a la realidad física, económica y social de las provincias, cantones y ciudades del país, con graves consecuencias: elevados costos de mantenimiento vial y de operación de vehículos. En las carreteras del país se observa con frecuencia la gran diferencia en costo que implica no intervenir a tiempo en una vía, dejando desarrollar su deterioro y XVII postergando su mantenimiento o rehabilitación.

El turismo ecológico o ecoturismo es un enfoque para las actividades turísticas en el cual se privilegia la preservación y la apreciación del medio tanto natural como cultural que acoge a los visitantes. Debido a su rápido crecimiento también se lo ve como subsector de la actividad turística. Este movimiento apareció a finales de la década de 1980, pero muy recientemente ha logrado despertar el interés internacional tanto como para que la ONU dedicara el año 2002 al turismo ecológico. La Sociedad 
Internacional de Ecoturismo (TIES) define Ecoturismo como "un viaje responsable a aéreas naturales que conservan el ambiente y mejoran el bienestar de la población local".

La forma como se organiza la sociedad para producir determinados bienes y servicios no se limita únicamente a los procesos estrictamente técnicos o económicos, sino que también tiene que ver con todo el conjunto de interacciones entre los distintos actores sociales que utilizan los recursos que tienen a su disposición para llevar adelante las actividades productivas. A ese conjunto, que incluye los productos, los procesos productivos y las relaciones sociales resultantes de esos procesos, se denomina matriz productiva 5.

La economía nacional se ha visto determinada por la diferencia existente entre los precios de productos primarios, que son aquellos que Ecuador exporta, y aquellos con mayor valor agregado que en cambio son aquellos bienes y servicios que el país importa; sumado a la variación de los precios internacionales de las materias primas. Es por esto que la economía ecuatoriana ha venido sufriendo un proceso de intercambio desigual, que en la actualidad se pretende mitigar. (Ministerio de Turismo, 2015)

Transformar la matriz productiva es uno de los retos más ambiciosos del país, el que permitirá al Ecuador superar el actual modelo de generación de riquezas: concentrador, excluyente y basado en recursos naturales, por un modelo democrático, incluyente y fundamentado en el conocimiento y las capacidades de las y los ecuatorianos.

El diseño de investigación se contempla de dos maneras:

Investigación exploratoria, este tipo de investigación se centra en encontrar una perspectiva general del problema, ya que ayuda a dividir el problema principal en sus causas y efectos; por otra parte, esta investigación se aplica cuando existen pocas fuentes de información precisas o estudios previos que permitan tener un criterio más acertado de lo que se investiga; por tal razón, se aplica esta investigación pues poco se conoce de proyectos donde se hayan implementado rutas turísticas ecológicas en la ciudad de Guayaquil. Así mismo de acuerdo al autor Namakforoos, estable que el propósito de esta investigación es la siguiente:

\# Formular problemas para estudios más precisos o para desarrollo de hipótesis.

\# Establecer prioridades para futuras investigaciones.

\# Recopilar información acerca de un problema que luego se dedica a un estudio especializado.

\# Aumentar el conocimiento del problema.

\# Aclarar conceptos.

Investigación Descriptiva, se complementa a la investigación exploratoria ya que mediante esta forma de estudio el investigador pretende describir las características o rasgos más importantes de ciertos grupos, en este caso los gustos y preferencias de la ciudadanía guayaquileña en relación a la ruta turística propuesta. Del mismo modo, para establecer estos rasgos, se emplearán fórmulas para el cálculo de una muestra especifica partiendo como población la ciudad de Guayaquil, 
finalmente se analizan estas características a través de porcentajes y gráficos estadísticos para realizar proyecciones, como por ejemplo el nivel de demanda que tendría el proyecto.

De igual forma, la investigación se centrará en los siguientes aspectos:

Investigación documental, se realizará consultas de documentos, tales como: libros, revistas, periódicos, registros, constituciones, etc., con el objetivo de recaudar información valedera que proporcione los respectivos respaldos bibliográficos para el desarrollo de la investigación.

Investigación de campo, también conocida como investigación directa, es la que se efectúa en el lugar y tiempo en que ocurren los fenómenos objeto de estudio. Para poner en práctica este tipo de investigación se hará uso de algunas técnicas de recogida de datos para lo cual es necesario acudir al lugar de los hechos, dichas técnicas son:

- Observación directa: Utilizada para identificar las diferentes especies de flora y fauna de los sitios escogidos para la ruta.

- Encuestas: Estarán conformadas por un cuestionario de preguntas cerradas y se las efectuará a turistas, tanto nacionales como extranjeros, en diferentes puntos estratégicos de la ciudad.

\section{RESULTADOS}

El grado de erosión dominante en el sendero fue bajo a moderado, en general se observa abundante hojarasca, alta cantidad de raíces finas, y un horizonte A entre 2 a $5 \mathrm{~cm}$ de espesor.

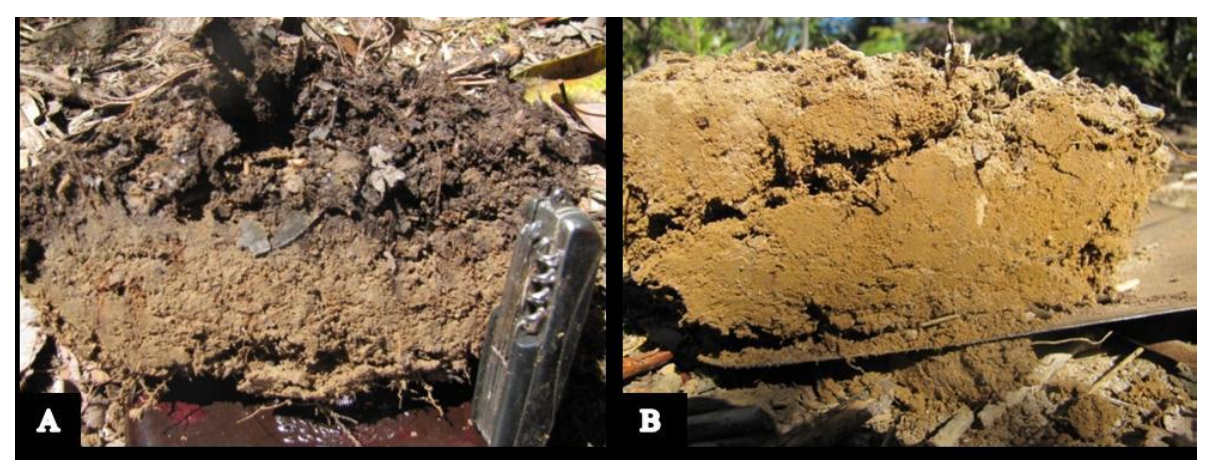

Figura 1

Nivel de erosión en la zona de estudio A) Erosión baja (tramo 1); B) erosión moderada (tramo 9). Los Autores

El nivel de anegamiento también fue bajo a moderado, presentándose zonas anegadas y con signos de anegamiento en las partes bajas y cercanas a cursos de agua. 


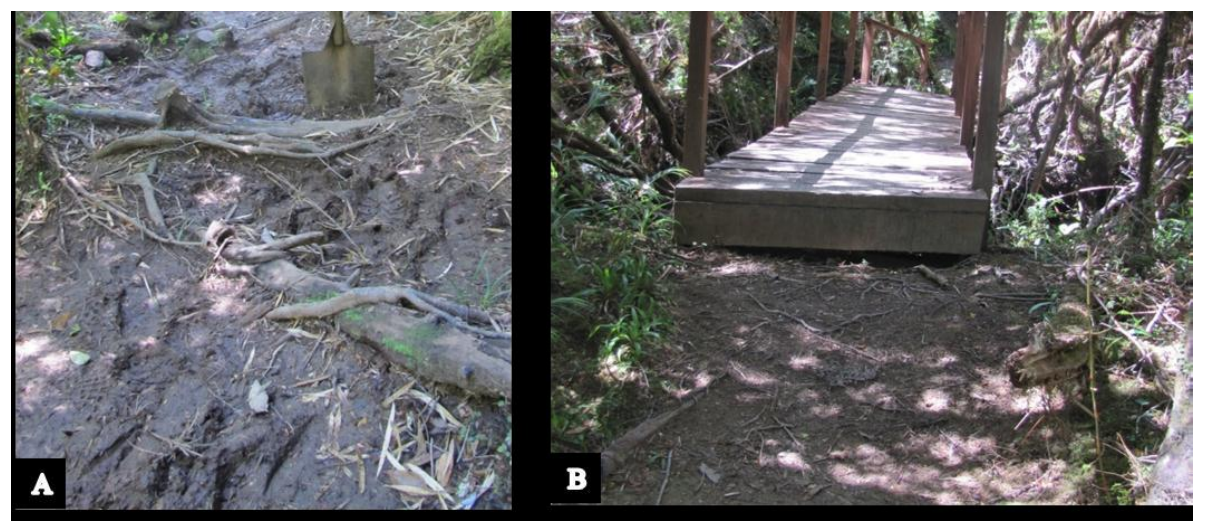

Figura 2.

Nivel de anegamiento en el sendero

A) Zona anegada (tramo 17); B) zona con evidencia de anegamiento (tramo 16).

Los autores

$\mathrm{Al}$ igual que los indicadores anteriores, el grado de alteración de la vegetación fue bajo a moderado, dado principalmente por la presencia de raíces expuestas.

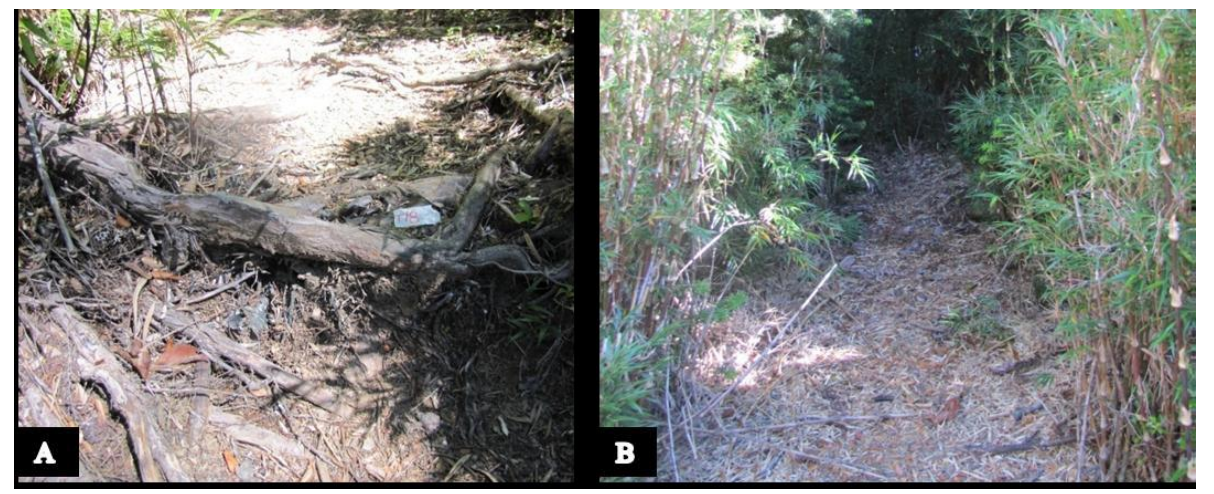

Figura 3.

Alteración de la vegetación en el sendero

A) moderada alteración; B) bajo nivel de alteración.

Los autores

Los tramos 12, 16 y 17 que pasan por esteros fueron los que presentaron mayores problemas de erosión, anegamiento y alteración de la vegetación. Adicionalmente, los tramos del 5 al 9 ubicados en la zona de renoval tuvieron un grado de erosión medio.

En todo el sendero no se registraron especies de aves sensibles a la presencia humana, y total ausencia de senderos informales.

Limitantes para el visitante

La pendiente y ancho de faja del sendero en general fue medianamente adecuada, con pendientes promedio sobre los 10 grados y un ancho de faja promedio de $60 \mathrm{~cm}$. Los tramos donde la pendiente promedio se mantuvo sobre 10 grados fueron desde el 9 al 18 , asociados principalmente a la presencia de quebradas. En cuanto al ancho de faja, los tramos que tuvieron mayor inestabilidad en su ancho, con un promedio de $50 \mathrm{~cm}$, fueron del 4 al 8. 


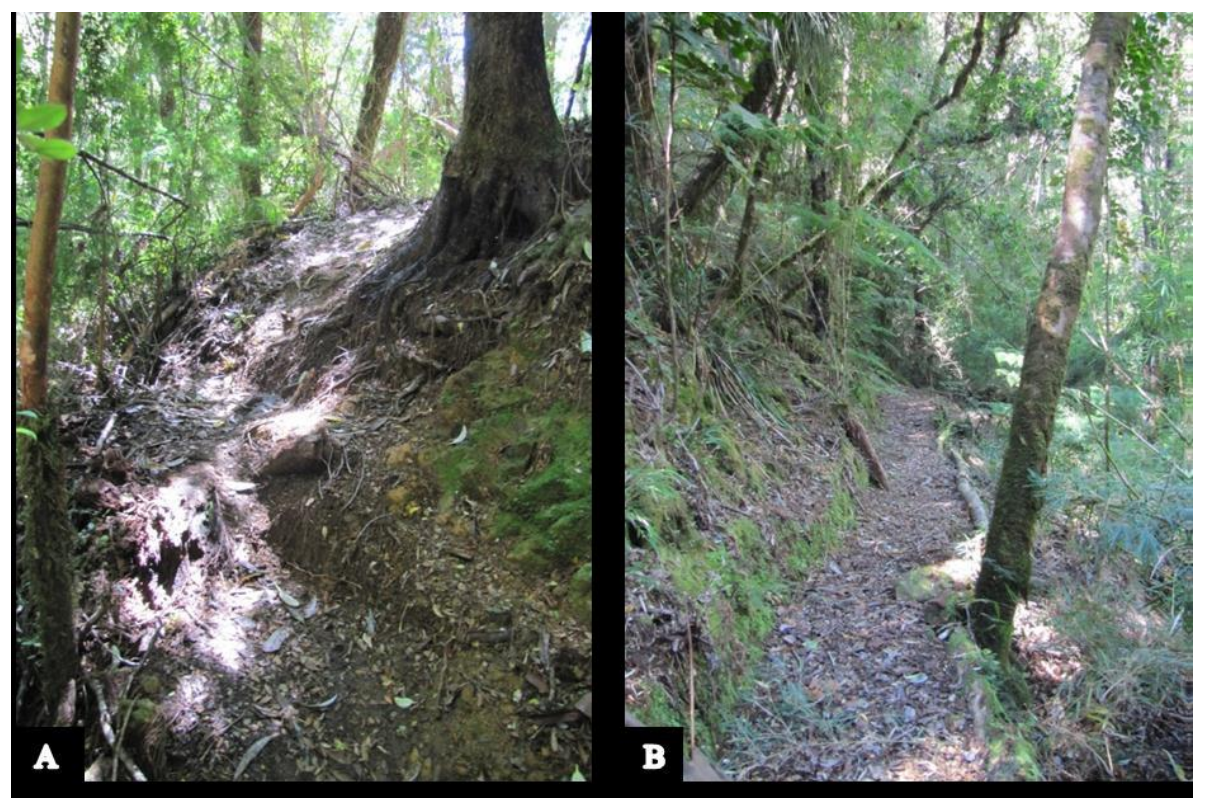

Figura 4.

Pendiente y ancho de faja predominante en el sendero

A) Pendiente y B) ancho de faja medianamente adecuada. Tramo 16 y 11 respectivamente.

Los autores

Los letreros de información e interpretación del sendero fueron retirados para su posterior restauración, por lo que se consideró como infraestructura menor en mal estado. En cuanto a la infraestructura mayor, el sendero esta implementado con portones en la entrada y salida, letreros de bienvenida y señalización, 8 puentes, escaleras y pasarelas en el sector del alerzal, y barandas de protección para el Alerce milenario. Los tramos que cuentan con mayor implementación son el 15 y 18, debido a la presencia de alerce 


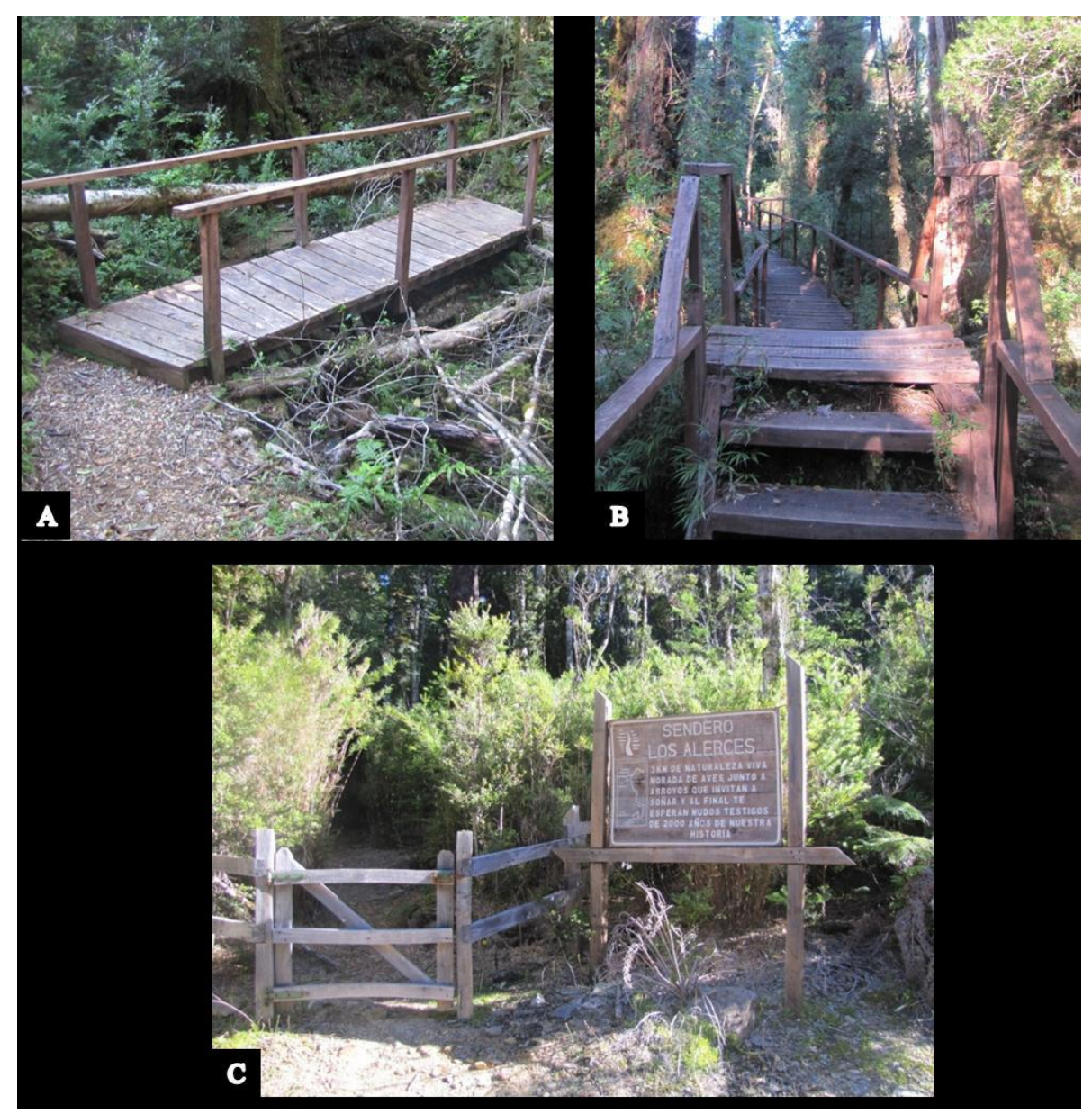

Figura 5

Infraestructura presente en el sendero

A) Puente sobre curso de agua; B) pasarela que protege la vegetación del alerzal,

Tramos 3 y 18 respectivamente. C) Infraestructura presente a la entrada al sendero. Los autores

Durante el recorrido del sendero no se encontraron animales molestos. Por otro lado, en relación a la accesibilidad, el sendero se encuentra a menos de un metro del camino principal, sin embargo, el estado de éste no es apto para el tránsito de todo vehículo, condición que empeora en la temporada invernal. 


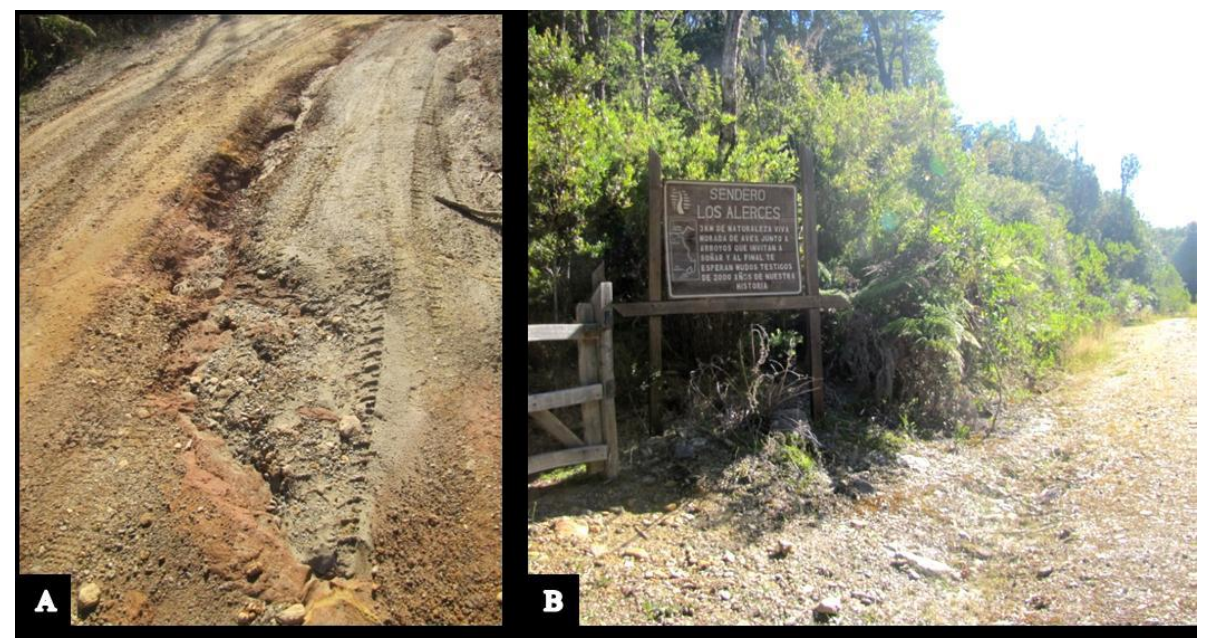

Figura 6.

Estado del camino hacia el sendero

A) Evidencia de grandes cárcavas en el camino Chaihuín - los alerces; B) vista lateral de la entrada al sendero Los autores

\section{DISCUSIÓN}

Los bosques de Alerce y Coigue presentes a lo largo del sendero Los Alerces mantienen una alta diversidad de flora y fauna endémica propia de los Bosques siempreverdes de la Cordillera Pelada (Luebert y Pliscoff 2004), sitio prioritario de conservación debido a su gran importancia como hábitat de especies endémicas, asociado a que durante las glaciaciones pleistocénicas fue refugio para muchas especies de flora y fauna (Vergara y Reyes 2000, Ramírez y San Martin 2006). Además, estos bosques se caracterizan por tener un dosel multiestratificado, con abundantes lianas, epífitas, árboles muertos en pie, claros, troncos caídos, denso sotobosque de quila y grandes árboles que emergen sobre el resto del dosel, que le otorgan alta heterogeneidad ambiental y complejidad estructural que permiten la continuidad de poblaciones de animales, principalmente aves y anfibios (Díaz 2005). Adicionalmente, las condiciones de estos ecosistemas boscosos determinan la variedad de texturas, colores y aromas presentes. En el sendero esto se aprecia principalmente en el cambio de bosque adulto a renoval, donde la composición y estructura del bosque producen una variación de luz, temperatura y humedad, así como en la composición de la flora y fauna, y el suelo, que ocasionan un alto contraste de sensaciones, colores, texturas y aromas para los visitantes.

Los aspectos físicos de la Cordillera de la Costa, como el clima, la geomorfología y el suelo son los componentes que determinan las limitantes del sendero. El clima templado lluvioso se caracteriza por una gran cantidad de precipitaciones durante el periodo invernal (Delgado 2010), condición que marca una fuerte estacionalidad y disminución en la visitación de las APs y la realización de actividades al aire libre como el senderismo, debido principalmente a que la lluvia, viento y frío disminuyen el confort del turista y el nivel de seguridad. Adicionalmente, 
con el clima varían la coloración de la vegetación, la posibilidad de avistamiento de fauna y flores silvestres, entre otras características, haciendo que la época de muestreo en que se realice un estudio de atractivo turístico varié considerablemente.

La geomorfología del lugar está representada por la escasa presencia de valles a lo largo de los cursos de agua y un relieve ligero a moderadamente ondulado con numerosas quebradas . Esta condición, sumada al tipo de suelo perteneciente a la serie Hueicoya (HEY), limitan al sendero por su moderada probabilidad de erosión y drenaje restringido, que provoca además condiciones de anegamiento . También, este tipo de suelo aumenta la degradación de raíces por pisoteo, principalmente de coigues cuyas raíces son superficiales. En relación a los métodos existentes para evaluar senderos es posible mencionar que métodos como la CC (Capacidad de Carga Turística), han sido ampliamente criticados debido a su carácter numérico y compleja aplicabilidad. López J. y López L. critican a estos estudios por su perspectiva numérica, y creen junto a Watson y Kopachevsky, que existe una falta de conciliación entre los componentes descriptivos y evaluativos que se proponen. Por otra parte, Alipour en López J. y López L., sugieren una serie de factores que han obstaculizado un mayor uso de la capacidad de carga turística, como: la falta de indicadores aceptables del entorno; la subjetividad de ciertos parámetros; y la complejidad de las técnicas utilizadas por los investigadores. A pesar de esto, López J. y López L. establecen que la CC sigue siendo uno de los instrumentos de medida más representativos del turismo sustentable.

El Sendero de Chile, aplica de cierta forma un estudio de capacidad de carga, pero su carácter es mayormente cualitativo, y no se establecen explícitamente características ambientales, topográficas y/o socio-culturales para diferenciar los tramos. La gran diferencia entre los estudios de CC con el método propuesto en este informe, es que éste no busca cuantificar cuanto puede resistir un sendero según su nivel de visitación, sino identificar las limitaciones o restricciones y el potencial del sendero para un mejor manejo y aprovechamiento de los recursos presentes. Por otra parte, el método descrito por Galacho y Arrebola propone un análisis a nivel de tramo logrando identificar los sitios más críticos, de forma similar al método propuesto en este informe. La ventaja del método de Galacho y Arrebola es principalmente el menor esfuerzo de muestreo. Sin embargo, el valor económico de las imágenes y licencias de los programas SIG pueden compensarse con los recursos requeridos para medir los 15 indicadores del método propuesto, principalmente si se lleva a una mayor escala de muestreo. Este método por sí solo no permite el análisis de variables cualitativas como diversidad, percepción, paisaje, entre otras, o para su incorporación se requiere de una exhaustiva base de datos difícil de conseguir.

En general, ningún método utilizado para evaluar senderos, abarca el nivel de detalle propuesto, componentes ecosistémicos, diversidad de flora y fauna, valor científico, valor paisajístico, accesibilidad, infraestructura, topografía, clima, criterios intrínsecos como percepción 
sensitiva y componentes culturales, todo de manera íntegra. Su carácter cualitativo y cuantitativo sugiere que puede ser una herramienta de alta aplicabilidad, lo cual fue demostrado en terreno. Siendo uno de los requerimientos para desarrollar este método la capacidad técnica para la toma de datos de terreno. El nivel de detalle, se traduce en el esfuerzo de muestreo por parte del evaluador. El posterior análisis de datos no presenta mayor complejidad, debido a que se sintetizan todos los datos obtenidos en una matriz cuantitativa, que luego se traduce en categorías cualitativas previamente descritas. Por lo antes expuesto, para el desarrollo del turismo sostenible y sustentable es de vital importancia el Sendero Turístico-Ecológico para la Comuna Palmar, Parroquia Colonche, Cantón Santa Elena.

\section{Referencias}

Andes Agencia publicitaria de noticias del Ecuador y Suramerica. (2013). Andes. Recuperado de http://www.andes.info.ec/es/turismo/gobierno-ecuadorapuesta-serio-desarrollo-turistico-cuadruplica-presupuesto-esesector.html

M.I. Municipalidad de Guayaquil. (2006). Ordenanza de Ordenamiento Territorial. Recuperado de https://www.mvotma.gub.uy/10aniversarioleyot/item/10011781-guias-metodologicas-de-ordenamiento-territorialy-desarrollo-sostenible-elaboracion-de-la-ordenanza-departamental

M.I. Municipalidad de Guayaquil. (2011). Reformas Ordenanza Sustitutivas de Parcelaciones y Desarrollo Urbanistico. Recuperado de http://guayaquil.gob.ec/Ordenanzas/Planificaci \%C3\%B3n\%20Urbana/14-1-2010.\%20Ordenanza\%20sustitutiva \%20de\%20parcelaciones\%20y\%20desarrollos\%20Urban \%C3\%ADsticos.\%20pdf.pdf

MINISTERIO DE TRANSPORTE Y OBRAS. (2013). PROCEDIMIENTOS PARA PROYECTOS VIALES. Recuperado de https://www.obraspublicas.gob.ec/wp-content/uploads/ downloads/2013/12/01-12-2013_Manual_NEVI-12_VOLUMEN_1.pdf

Ministerio de Turismo del Ecuador. (2013). Informe de Gestión. Recuperado de https://www.planificacion.gob.ec/wp-content/uploads/ downloads/2014/04/INFORME-FINAL-ZONA-9MATRIZ-29-04-141.pdf

Ruiz Ballesteros, E., \& Solis Carrión, D. (2007). Turismo Comunitario en Ecuador. Ecuador: Abya Yala. 